\title{
Long-Term Patency of Visceral Vessels After Thoracoabdominal Aortic Repair
}

\author{
Andrea Kahlberg, Vincenzo Ardita, \\ Angela M. R. Ferrante, Luca Bertoglio, \\ Daniele Mascia, Domenico Baccellieri, \\ Germano Melissano, and Roberto Chiesa
}

\subsection{Introduction}

Open repair of thoracoabdominal aortic aneurysms (TAAAs) is an extraordinary challenge for both vascular surgeons and patients. Consistent outcome data have been reported by highvolume centers with 30-day mortality rates lower than $10 \%$ and improved long-term survivals [1]. The development of devices and techniques have allowed total endovascular treatment of TAAA with fenestrated (FEVAR) or branched (BEVAR) endografts [2-6]. Early results are encouraging;

\footnotetext{
A. Kahlberg $(\bowtie) \cdot$ V. Ardita $\cdot$ L. Bertoglio $\cdot$ D. Mascia G. Melissano Vascular Surgery, Vita-Salute University School of Medicine, San Raffaele Scientific Institute, Milan, Italy

e-mail: kahlberg.andrea@hsr.it; ardita.vincenzo@hsr.it; bertoglio.luca@hsr.it; mascia.daniele@hsr.it; melissano.germano@hsr.it
}

\author{
A. M. R. Ferrante \\ Unit of Vascular Surgery, Fondazione Policlinico \\ Universitario A. Gemelli IRCCS, Università Cattolica \\ del Sacro Cuore, Rome, Italy \\ e-mail: Angela.Ferrante@unicatt.it \\ D. Baccellieri \\ Unit of Vascular Surgery, San Raffaele Scientific \\ Institute, Milan, Italy \\ e-mail: baccellieri.domenico@hsr.it \\ R. Chiesa \\ Unit of Vascular Surgery, San Raffaele Scientific \\ Institute, Vita-Salute University, Milan, Italy \\ e-mail: chiesa.roberto@hsr.it
}

a recent meta-analysis has reported a 30-day mortality rate of $8.6 \%$, spinal cord ischemia in $17.3 \%$ of cases with permanent consequences in $4.9 \%$, and renal failure in $25.4 \%$. However, anatomic feasibility of BEVAR/FEVAR is still limited, manufacturing times for custom devices are lengthy, and open repair remains the only viable strategy in a number of specific conditions, such as emergency cases, infections, and patients affected by connective tissue disorders.

Recent publications on FEVAR/BEVAR series have highlighted the fate of revascularized visceral vessels (VVs), reporting a progressive loss of stented visceral branches [7]. In addition, a comparison of long-term results with those observed in conventional open repair is still unachievable for such recent technology, which is currently performed in few specialized centers on relatively small groups of patients.

Surprisingly, few data have been reported in the recent literature about the late fate of visceral arteries after open repair of TAAAs [8, 9]. In 2010, Kulik et al. [10] reviewed their TAAA repair experience using a presewn aortic branched graft. Between March 2003 and December 2008, 52 patients with Crawford extent II and III TAAAs underwent surgical repair using a presewn aortic branched graft. The patency rate of the branches to the visceral and renal arteries was $100 \%$ and $98 \%$, at 1 and 5 years, respectively. Of the 148 graft branches, two became occluded and four developed stenosis (two patients). One patient 
required percutaneous stenting of three stenosed branches, and one patient died after acute occlusion of two branches and stenosis of a third. During the follow-up period that extended to 6.3 years, there were ten late deaths. Six patients required reoperation on the aortic graft or contiguous aorta, but no reoperations were required on the visceral abdominal aorta or its branches. The authors concluded that the use of a presewn aortic branched graft is a safe and suitable option for TAAA repair. Another study performed by Shahverdyan et al. [11], in 2013, showed outcomes of long-term bypass graft patency after visceral hybrid repair of thoracoabdominal aortic aneurysms (TAAAs). Between July 2004 and September 2012, 46 patients (67.5 years in median; range 35-88) with TAAAs underwent to the visceral hybrid procedure (164 grafts). The inflow sites of visceral revascularization originated either from the native artery or from aortic prosthetic grafts. The 30-day primary patency of all grafts was $87.9 \%$. The long-term patency remained $87.9 \pm 2.7 \%, 86.1 \pm 3.2 \%$, and $86.1 \pm 3.2 \%$ after 1,3 , and 5 years, respectively. The patency of all hepatic grafts was $100 \%$. The patency of grafts to the superior mesenteric artery was $88.8 \pm 4.8 \%$ after 1,3 , and 5 years. The patency was $87.2 \pm 6 \%, 87.2 \pm 6 \%$, and $87.2 \pm 6 \%$ for the left renal artery and $76 \pm 7 \%, 69.6 \pm 8.8 \%$, and $69.6 \pm 8.8 \%$ for the right renal artery after 1,3 , and 5 years, respectively. The authors concluded that hybrid repair demonstrates excellent results regarding long-term bypass graft patency of renovisceral arteries except for the right renal artery. In 2015 Youssef et al. [9] showed data of 62 patients (mean age is $66 \pm 10$ years, 40 men) who had been operated on for TAAA of Crawford types I (8), II (13), III (13), and IV (24) and Safi type V (4). A total of 181 vessels were revascularized by either patch inclusion $(n=147)$ or selective revascularization (bypass or transposition, $n=34$ ); 48 survived the procedure, resulting in a number of vessels available for follow-up of 154 (patch, 126; selective revascularization, 28). The patency rates for overall, patch, and selective revascularization were $95.2 \%, 94.2 \%$, and $100 \%$ at 5 years and $83.7 \%, 81.3 \%$, and $100 \%$ at 10 years, respectively. The patency rates for the celiac trunk, superior mesenteric artery, and left and right renal artery were $100 \%, 97.5 \%, 92.3 \%$, and $90.3 \%$ at 5 years. More recently, Kouchoukos et al. [12] showed their experience of 298 branch grafts to the celiac, superior mesenteric, and renal arteries performed in a total of 99 of 130 patients undergoing open thoracoabdominal aortic aneurysm repair. The authors showed a freedom from occlusion of $98 \%, 97 \%$, and $93 \%$, at 1,5 , and 10 years of follow-up, respectively.

\subsection{San Raffaele Hospital Experience}

Between January 2009 and July 2015, 382 patients underwent open TAAA repair at our institution. Patients were selected from a prospectively maintained computerized database and retrospectively reviewed. Demographics and perioperative risk factors are reported in Table 20.1.

An adequate preoperative assessment of physiological reserve of cardiac, pulmonary, and renal function was used to evaluate operative risks, to plan the best operative strategy, and to consider additional perioperative organ protection strategies. A coronary computed tomography was performed in selected patients with a history of coronary artery disease from 2009 to 2012 and in all elective patients since 2013. A preoperative study was conducted on all patients by a computed tomography angiography (CTA) scan of the thoracoabdominal aorta.

The anatomical extent of the aneurysm was defined according to the Crawford classification [13]. Chronic obstructive pulmonary disease (COPD) included all patients with a grade $\geq 2$ in the GOLD score system [14]. Patients were stratified by chronic kidney disease (CKD) stages according to the NKF-KDOQI (National Kidney Foundation's Kidney Disease Outcomes Quality Initiative) guidelines [15]. Cerebrovascular disease included all cases with a history of TIA, minor or major stroke, or carotid occlusive disease.

Details of the surgical technique for open repair of TAAAs at our institution have been previously described [12]. The strategy for VV revascularization was customized (Fig. 20.1) according to the following principles: Inclusion in a "beveled" aortic anastomosis or in Carrel patch was favored in 
Table 20.1 Demographics and preoperative risk factors of 382 TAAA patients

\begin{tabular}{|c|c|c|c|}
\hline Variable & Definition & No. & $(\%)$ \\
\hline Age & Years (mean $\pm \mathrm{SD})$ & $66 \pm 10$ & \\
\hline Gender & Male & 284 & $(74 \%)$ \\
\hline Coronary artery disease & Yes & 76 & $(20 \%)$ \\
\hline COPD & Yes & 134 & $(35 \%)$ \\
\hline History of smoking & Yes & 186 & $(49 \%)$ \\
\hline Hypertension & Yes & 302 & $(79 \%)$ \\
\hline Diabetes mellitus & Yes & 28 & $(7 \%)$ \\
\hline Dyslipidemia & Yes & 96 & $(25 \%)$ \\
\hline Cerebrovascular disease & Yes & 114 & $(30 \%)$ \\
\hline \multirow[t]{5}{*}{ Renal function } & CKD stage 1 & 207 & $(54 \%)$ \\
\hline & CKD stage 2 & 119 & $(31 \%)$ \\
\hline & CKD stage 3 & 43 & $(11 \%)$ \\
\hline & CKD stage 4 & 10 & $(3 \%)$ \\
\hline & CKD stage 5 & 3 & $(1 \%)$ \\
\hline \multirow[t]{4}{*}{ TAAA extent } & Type I & 78 & $(20 \%)$ \\
\hline & Type II & 122 & $(32 \%)$ \\
\hline & Type III & 103 & $(27 \%)$ \\
\hline & Type IV & 79 & $(21 \%)$ \\
\hline TAAA maximum diameter & $\mathrm{mm}($ mean $\pm \mathrm{SD})$ & $66 \pm 13$ & \\
\hline \multirow[t]{3}{*}{ Etiology } & Atherosclerosis & 312 & $(82 \%)$ \\
\hline & Dissection & 46 & $(12 \%)$ \\
\hline & Connective tissue disorder & 24 & $(6 \%)$ \\
\hline \multirow[t]{3}{*}{ Previous surgery } & TAA open & 19 & $(5 \%)$ \\
\hline & TEVAR & 33 & $(9 \%)$ \\
\hline & AAA & 64 & $(17 \%)$ \\
\hline \multirow[t]{2}{*}{ Presentation } & Symptomatic & 65 & $(17 \%)$ \\
\hline & Ruptured & 12 & $(3 \%)$ \\
\hline
\end{tabular}

$T A A A$ thoracoabdominal aortic aneurysm, $C O P D$ chronic obstructive pulmonary disease, $C K D$ chronic kidney disease, $T A A$ thoracic aortic aneurysm, TEVAR thoracic endovascular aortic repair, $A A A$ abdominal aortic aneurysm

case of short distance between the VV ostia; when the aneurysm included the visceral aortic segment, no more than three VVs were normally reimplanted together in a single Carrel patch to avoid the risk of late aneurysm recurrence [16]. A VV originating far from the others was usually reconstructed separately by direct reimplantation on the main aortic graft or by individual graft interposition (6-mm heparin-bonded Dacron or ePTFE). Since 2012, the Gore Hybrid Vascular Graft (W.L. Gore and Associates, Flagstaff, AZ) has been selectively used to perform separate revascularization of one or two VVs allowing for distal sutureless anastomosis. The specific operative technique for this device has been previously described in detail [12]. A separate revascularization of all VVs has always been preferred-in most cases accomplished with presewn multibranched grafts (Coselli graft, Vascutek Terumo, Renfrewshire, UK, or Hemashield
Platinum TAAA graft, Maquet, Sunderland, UK) - in case of large aneurysms with excessive distance between all VV ostia, in the event of recurrent aneurysmal disease after previous TAAA surgery, or in patients affected by connective tissue disorders.

Intraoperative details included techniques used for VV revascularization and postoperative outcome variables. The follow-up protocol included a physical exam and CTA at 3 and 12 months postoperatively and yearly thereafter. Patients unable to comply with the follow-up protocol were contacted by phone interviews and asked to provide digital supports of their imaging studies when available. Patients not responding to two consecutive attempts of phone interviews were considered lost to follow-up. Restenosis was defined as $>50 \%$ reduction of the vessel lumen on both arterial and late-phase scans. 

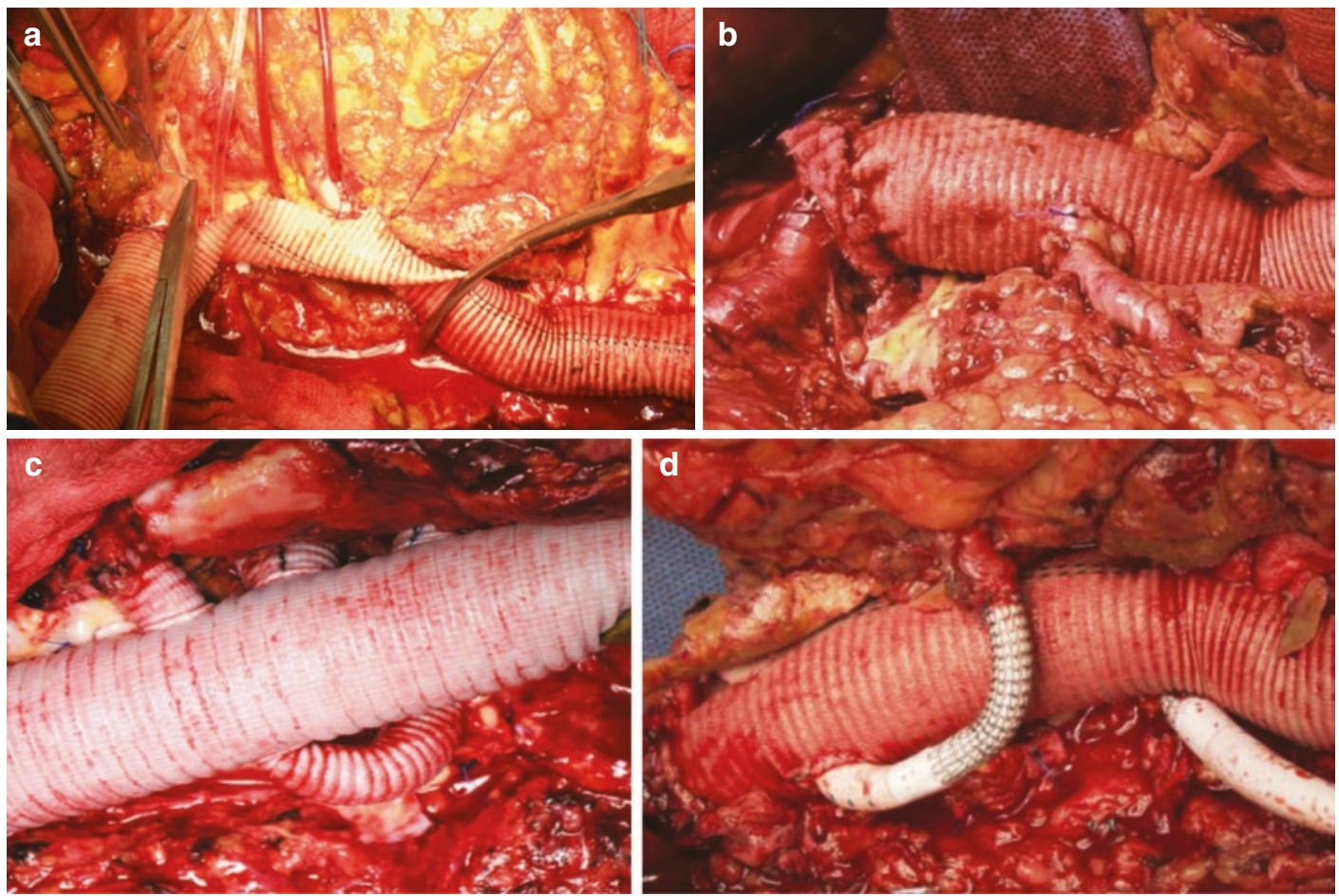

Fig. 20.1 Intraoperative views of different techniques for VV reconstruction: (a) Carrel patch including CT (C), SMA (M), and RRA (R). (b) Direct reimplantation of the LRA on the main aortic graft. (c) Revascularization of all
VVs using a presewn multibranched graft. (d) Bilateral renal artery revascularization using two Gore Hybrid Vascular Grafts with sutureless distal anastomosis

Table 20.2 Techniques used for reconstruction of visceral vessels in 382 TAAA patients

\begin{tabular}{l|c|c|c|c}
\hline & CT & SMA & RRA & LRA \\
\hline Inclusion (Carrel patch/beveled anast.) & 321 & 349 & 319 & 175 \\
\hline Direct reimplantation & 1 & 0 & 0 & 18 \\
\hline Single bypass (conventional) & 5 & 2 & 6 & 67 \\
\hline Single bypass (sutureless) & 5 & 3 & 21 & 91 \\
\hline Multibranched graft & 26 & 25 & 20 & 22 \\
\hline Associated endarterectomy & 3 & 1 & 10 & 30 \\
\hline Associated direct stenting & 9 & 4 & 19 & 37
\end{tabular}

$C T$ celiac trunk, SMA superior mesenteric artery, $R R A$ right renal artery, $L R A$ left renal artery

Cerebrospinal fluid drainage (CSFD) was used in 343 patients, and left heart bypass (LHBP) in 332. Overall, revascularization involved 1476 VVs: 358 celiac trunks (CT), 379 superior mesenteric arteries (SMA), 366 right renal arteries (RRA), and 373 left renal arteries (LRA). Techniques of VV reconstruction and associated procedures —endarterectomy or stenting - are reported in Table 20.2.
Perioperative results are summarized in Table 20.3. The in-hospital mortality was $7.6 \%$ (29 patients), including five intraoperative deaths. Complications occurred in $26.8 \%$ of patients $(n=102)$. Among 353 early-surviving patients, 15 were lost to follow-up. The mean length of follow-up was $22 \pm 17$ months.

General survival was $87 \%$ at 1 year, $84 \%$ at 2 years, $75 \%$ at 3 years, and $65 \%$ at 5 years 
(Fig. 20.2). No instances of bowel ischemia were recorded during follow-up. Eight patients developed late- onset renal failure requiring dialysis. Estimates for reintervention on VVs were 1.2\%, $6.3 \%$, and $17 \%$ at 1,3 , and 5 years, respectively (Fig. 20.3). All patients requiring reinterventions on VVs presented with asymptomatic restenosis and were treated by means of percutaneous stenting.

Of 338 patients available at follow-up, 247 had adequate imaging studies for analysis of $\mathrm{VV}$ patency. Among these, $952 \mathrm{VVs}$ were analyzed

Table 20.3 Perioperative results in 382 TAAA patients

\begin{tabular}{l|l|l}
\hline Variables & No. & $(\%)$ \\
\hline Intraoperative mortality & 5 & $(1.3 \%)$ \\
\hline In-hospital mortality & 29 & $(7.6 \%)$ \\
\hline ICU stay (hours, median, IQR) & $23(18-62)$ & \\
\hline LOS (days, mean \pm SD) & $11 \pm 9$ & \\
\hline Paraparesis & 12 & $(3.1 \%)$ \\
\hline Paraplegia & 19 & $(5.0 \%)$ \\
\hline Cerebral hemorrhage & 5 & $(1.3 \%)$ \\
\hline Stroke & 6 & $(1.6 \%)$ \\
\hline $\begin{array}{l}\text { Bleeding requiring } \\
\text { reintervention }\end{array}$ & 9 & $(2.4 \%)$ \\
\hline Bowel ischemia & & \\
\hline Renal failure requiring dialysis & 14 & $(1.6 \%)$ \\
\hline Respiratory failure & 57 & $(15 \%)$ \\
\hline Cardiac complications & 18 & $(4.7 \%)$ \\
\hline ICU intensive care unit, LOS & \multicolumn{1}{|c|}{6} \\
\hline
\end{tabular}

$I C U$ intensive care unit, $L O S$ length of hospital stay
(229 CT, 245 SMA, 238 RRA, and 240 LRA). Overall patency of analyzed VVs was $98 \%$ at 1 year, $95 \%$ at 3 years, and $94 \%$ at 5 years. An analysis of available images provided the following patency rate of individual vessels at 1, 3, and 5 years: $99 \%, 98 \%$, and $98 \%$ for CT; $100 \%$, $100 \%$, and $100 \%$ for SMA; $100 \%, 96 \%$, and $96 \%$ for RRA; and $91 \%, 87 \%$, and $82 \%$ for LRA (Fig. 20.4a). Compared with other VVs, the LRA showed a trend of significantly reduced patency in time (log rank [Mantel-Cox] test, $P<0.0001$ ). Freedom from restenosis or occlusion at 1,3 , and 5 years was $98 \%, 94 \%$, and $94 \%$ for CT; $100 \%$, $99 \%$, and $99 \%$ for SMA; $99 \%, 93 \%$, and $90 \%$ for RRA; and 91\%, 82\%, and 77\% for LRA ( $\log$ rank [Mantel-Cox] test, $P<0.0001$, Fig. 20.4b).

The group of 247 patients with available follow-up imaging was divided into three subgroups according to the VV reconstruction technique: patients with all VVs included in Carrel patch or in a "beveled" anastomosis (Group A, 94 patients), patients with 2 or 3 VVs included in Carrel patch and 1 separately reimplanted (Group B, 127 patients), and patients with all VVs reimplanted with separate revascularization (Group C, 26 patients). Freedom from any VV occlusion at 1 and 3 years was $95 \%$ and $87 \%$ for Group A, $89 \%$ and $79 \%$ for Group B, and $92 \%$ and $92 \%$ for Group C $(P=0.13$, Fig. 20.5).
Fig. 20.2 Kaplan-Meier curve of overall survival in 338 patients

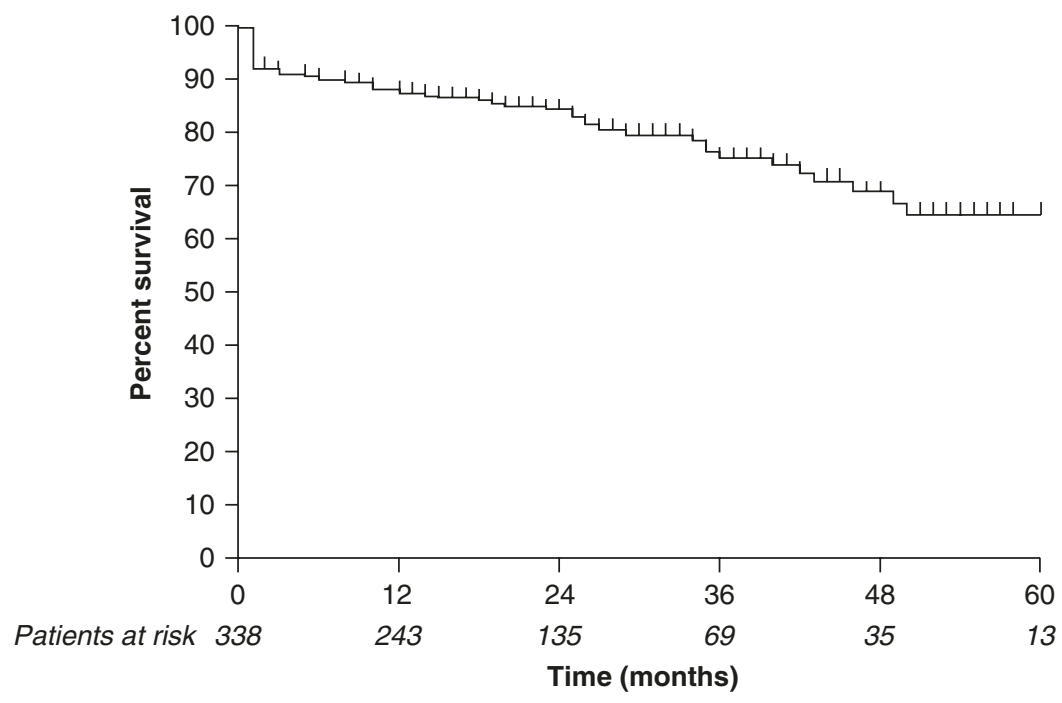


Fig. 20.3 Kaplan-Meier curve of freedom from reinterventions on VVs in 338 patients

Fig. 20.4 Kaplan-Meier curves of (a) VV patency and (b) VV freedom from restenosis/occlusion in 247 patients
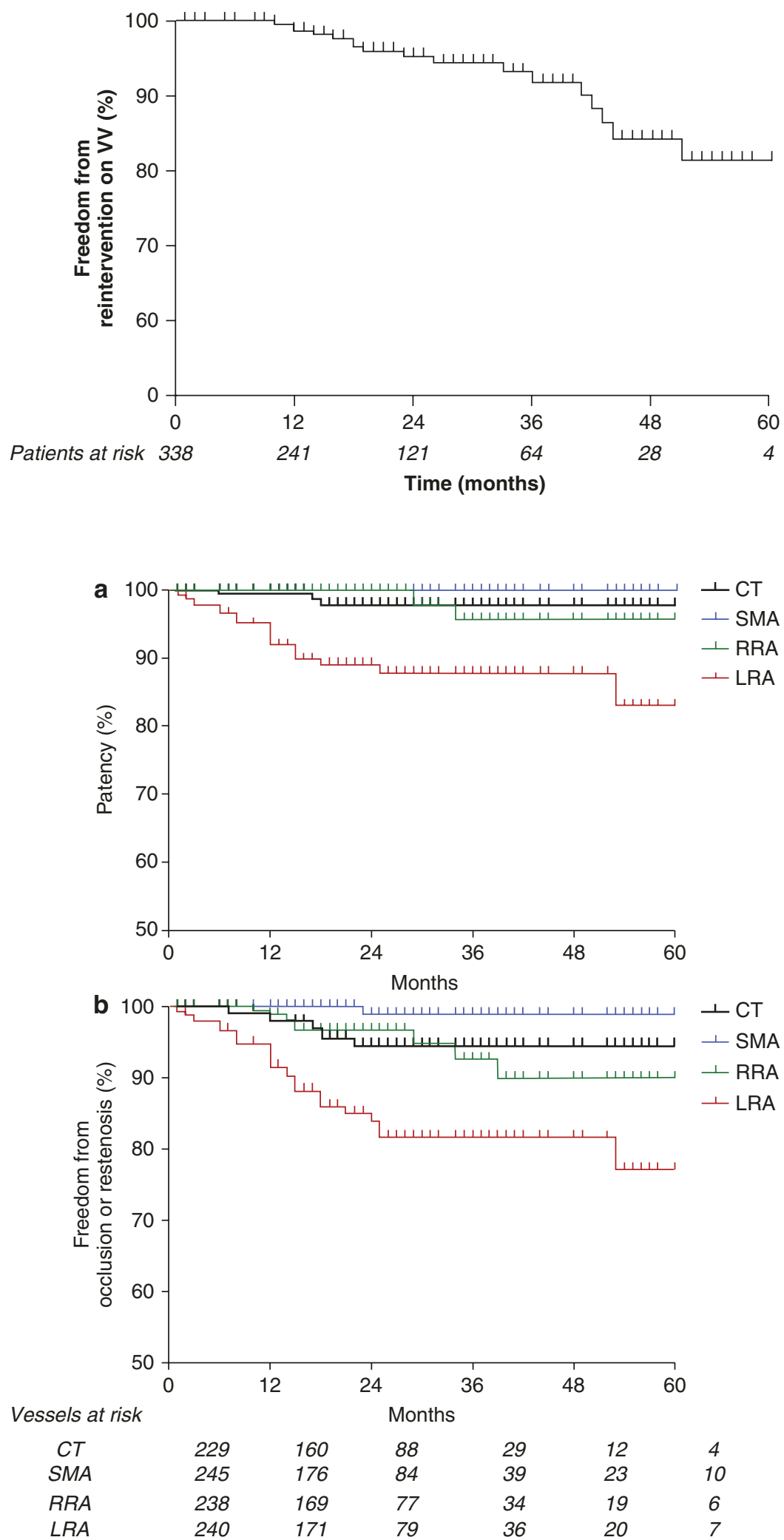
Fig. 20.5 Kaplan-Meier curves of freedom from any VV occlusion based on reconstructive techniques in 247 patients



Patients at risk

Group 1

Group 2

Group 3
75

91

11
Months

\section{8}

\section{3}

38

5
13

10 1

\subsection{Discussion}

Open repair has historically been considered the gold standard for treatment of TAAAs. Due to severe surgical trauma and early complication rates-even in experienced hands-total endovascular repair and hybrid techniques have been proposed as alternative options in patients considered at unacceptable high risk for conventional surgery.

Hybrid approach (surgical debranching of the abdominal aorta with retrograde bypass and stent grafting) has been proposed as an alternative option to patients who are unfit for surgery [15]. Although such a procedure avoids thoracolaparotomy, single-lung ventilation, aortic crossclamping, and extracorporeal perfusion, early results of several series reported mortality rates ranging between $2.3 \%$ and $24 \%[13,14]$ with significant morbidity (spinal cord ischemia up $8.4 \%$ and renal failure requiring dialysis up to $26.2 \%$ ) [13]. Moreover, the obstruction of visceral bypass is a relevant event in the midterm, affecting almost one third of cases with heavy clinical consequences, namely, emergency reintervention for bowel ischemia or permanent dialysis [13].

In recent years, the development of devices and techniques have allowed total endovascular treatment of TAAA with fenestrated (FEVAR) or branched (BEVAR) endografts [17, 18]. Early results are encouraging; a recent meta-analysis has reported a 30-day mortality rate of $8.6 \%$, spinal cord ischemia in $17.3 \%$ of cases with permanent consequences in $4.9 \%$, and renal failure in $25.4 \%$ [18].

However, anatomic feasibility of BEVAR/ FEVAR is still limited, manufacturing times for custom devices are lengthy, and open repair remains the only viable strategy in a number of specific conditions, such as emergency cases, infections, and patients affected by connective tissue disorders. Moreover, conventional surgery has yielded durable results, and a reliable comparison with long-term results of such recent endovascular techniques is still unachievable [19-22].

Moreover, although total endovascular repair has been performed since 2005 and there are centers with vast experience, long-term data about target vessel patency are still lacking. With a composite end point of branch instability (occlusion, migration, need for secondary intervention), results from the Cleveland Clinic have shown a rate of $70 \%$ at 5 years after fenestrated and branched endovascular repair of TAAA [23]. For hybrid TAAA repair, patency rates reported are in the range of $97 \%$ at 1 year [15] and $96 \%$ 
at 3 years [24]. Bianchini Massoni et al. performed a multicenter study providing patency data extending up to 4 years, and there, a patency of $79 \%$ was noted [19].

Our findings confirm that, in high-volume centers, perioperative mortality and morbidity rates are acceptable, and open surgery results are durable with high survival rates also at midterm follow-up.

The reconstruction strategy of VV represents a critical point in TAAA surgical repair, often requiring complex technical skills. Early failure of reconstructed branches may result in catastrophic outcomes. A number of techniques for VV reconstruction were described in the past by several authors. Inclusion in a visceral aortic patch (VAP) is the most commonly used strategy to obtain simultaneous revascularizations of multiple VVs, decreasing the global number of anastomoses. Separate reattachment is frequently required when VV ostia are distant, and this particularly applies to the LRA. Separate reattachment of VVs may be performed by direct reimplantation on the main aortic graft or by graft interposition. Recently, we have reported the use of "hybrid" grafts allowing to perform distal sutureless anastomosis as a useful tool for VV revascularization during TAAA open repair [25]. Alternatively, the use of presewn aortic branched grafts allows to separately revascularize each $\mathrm{VV}$, and, for this reason, it is considered the preferred choice in most young patients affected by connective tissue disorders [10]. There are very few data available from the literature about the fate of reconstructed VV and the possible effects of the surgical technique used. Our overall late patency results (94\% at 5 years) favorably compare with other series reporting a total patency of the reimplanted vessels of $95.4 \%$ and $97 \%$ at 5 years $[8,9]$.

In our experience, late patency rates were not apparently biased by the technique adopted. Interestingly, our study showed that the LRA has a significantly lower patency at all time points as compared to the other VVs. The cause of this finding is not clear, but it can be hypothesized that the intraoperative visceral rotation and subsequent repositioning may play a crucial role leading to kinking or angulation of the revascularized artery.
Moreover, the LRA was most often involved by adjunctive procedures, such as endarterectomy or direct stenting.

Late patency of visceral branches following total endovascular TAAA repair (FEVAR and/ or BEVAR) is an open issue. Different groups reported excellent early patency rates, followed in the midterm by a slow but progressive loss of visceral branches [1, 26, 27]. Late occlusion involves VVs at different rates, with a significant prevalence of renal arteries compared to other vessels. Renal branch occlusion has been observed in $8-9.6 \%$ of cases at midterm follow-up [7, 28]. Along with the high individual anatomic variability of the renal artery angle compared with other VVs, additional factors-e.g., respiratory motion and small vessel size - could have an adjunctive stress effect on stents.

The possible role of different devices employed in VV endovascular reconstruction has not been confirmed in a multicentric series [7], while the mean artery length and anatomic location were considered more likely associated with a poor branch outcome, and this finding could be a touchpoint with possible mechanical factors influencing observed late fate of LRA after open repair-particularly when presewn grafts are used [28].

Further studies are required to evaluate long-term survival and patency rates of visceral branches after total endovascular TAAA repair. Our series of 382 consecutive patients treated with open surgical repair provided early results in line with other high-volume centers and remarkable late patency rates for VV reconstruction. Based on a group of 247 patients and $952 \mathrm{VV}$ available for imaging study, this is, to the best of our knowledge, the largest series of surgical TAAA patients with long-term imaging evaluation of VV. This could be a useful benchmark for comparisons with future endovascular results.

\section{References}

1. Hu Z, Li Y, Peng R, Liu J, Jia X, Liu X, et al. Multibranched stent-grafts for the treatment of thoracoabdominal aortic aneurysms: a systematic review and meta-analysis. J Endovasc Ther. 2016;23:626-33. 
2. Coselli JS, LeMaire SA, Preventza O, de la Cruz KI, Cooley DA, Price MD, et al. Outcomes of 3309 thoracoabdominal aortic aneurysm repairs. J Thorac Cardiovasc Surg. 2016;151:1323-38.

3. Amiot S, Haulon S, Becquemin JP, Magnan PE, Lermusiaux P, Goueffic Y, et al. Fenestrated endovascular grafting: the French multicentre experience. Eur J Vasc Endovasc Surg. 2010;39:537-4.

4. Mastracci TM, Eagleton MJ, Kuramochi Y, Bathurst S, Wolski K. Twelve-year results of fenestrated endografts for juxtarenal and group IV thoracoabdominal aneurysm. J Vasc Surg. 2015;61:355-64.

5. Dias NV, Sonesson B, Kristmundsson T, Holm H, Resch T. Short-term outcome of spinal cord ischemia after endovascular repair of thoracoabdominal aortic aneurysms. Eur J Vasc Endovasc Surg. 2015;49:403-9.

6. Verhoeven ELG, Katsargyris A, Bekkema F, Oikonomou K, Zeebregts CJ, Ritter W, et al. Editor's choice - ten-year experience with endovascular repair of thoracoabdominal aortic aneurysms: results from 166 consecutive patients. Eur J Vasc Endovasc Surg. 2015;49:524-31.

7. Verzini F, Loschi D, De Rango P, Ferrer C, Simonte $\mathrm{G}$, Coscarella C, et al. Current results of total endovascular repair of thoracoabdominal aortic aneurysms. J Cardiovasc Surg. 2014;55:9-19.

8. Mastracci TM, Carrell T, Constantinou J, Dias N, Martin-Gonzales T, Katsargyris A, et al. Editor's choice - effect of branch stent choice on branchrelated outcomes in complex aortic repair. Eur J Vasc Endovasc Surg. 2016;51:536-42.

9. Youssef M, Neufang A, Jungmann F, Vahl C-F, Doweiler B. Patency of renal and visceral vessels after open thoracoabdominal aortic replacement. J Vasc Surg. 2015;62:594-9.

10. Kulik A, Kastner CF, Kouchoukos NT. Patency and durability of presewn multiple branched graft for thoracoabdominal aortic aneurysm repair. J Vasc Surg. 2010;51:1367-72.

11. Shahverdyan R, Gawenda M, Brunkwall J. Five year patency rates of renal and visceral bypasses after abdominal debranching for thoraco-abdominal aortic aneurysms. Eur J Vasc Endovasc Surg. 2013;45(6):648-56.

12. Kouchoukos NT, Kulik A, Castner C. Branch graft patency after open repair of thoracoabdominal aortic aneurysms. J Thorac Cardiovasc Surg. 2017;153:S14.

13. Chiesa R, Kahlberg A, Mascia D, Tshomba Y, Civilini E, Melissano G. Use of a novel hybrid vascular graft for sutureless revascularization of the renal arteries during open thoracoabdominal aortic aneurysm repair. J Vasc Surg. 2014;60:622-30.

14. Crawford ES, Crawford JL, Safi HJ, Coselli JS, Hess KK, Brooks B, et al. Thoracoabdominal aortic aneurysms: preoperative and intraoperative factors determining immediate and long-term results of operations in 605 patients. J Vasc Surg. 1986;3:389-404.

15. National Kidney Foundation. K/DOQI clinical practice guidelines for chronic kidney disease: evaluation, classification, and stratification. Am J Kidney Dis. 2002;39(suppl.1):S1-266.

16. Chiesa R, Melissano G, Civilini E, Bertoglio L, Rinaldi E, Marone EM, et al. Video-atlas of open thoracoabdominal aortic aneurysm repair. Ann Cardiothorac Surg. 2012;1:398-403.

17. Quinones-Baldrich WJ, Panetta TF, Vescera CL, Kashyap VS. Repair of type IV thoracoabdominal aneurysm with combined endovascular and surgical approach. J Vasc Surg. 1999;30:555-60.

18. Lin PH, Kougias P, Bechara CF, Wakley SM, Bakaeen FG, Lemaire SA, et al. Clinical outcome of staged versus combined treatment approach of hybrid repair of thoracoabdominal aortic aneurysm with visceral vessel debranching and aortic endograft exclusion. Perspect Vasc Surg Endovasc Ther. 2012;24:5-13.

19. Bianchini Massoni C, Geisbüsch P, Gallitto E, Hakimi M, Gargiulo M, Böckler D. Follow-up outcomes of hybrid procedures for thoracoabdominal aortic pathologies with special focus on graft patency and late mortality. J Vasc Surg. 2014;59:1265-73.

20. Verhoeven EL, Tielliu IF, Bos WT, Zeebregts CJ. Present and future of branched stent grafts in thoracoabdominal aortic aneurysm repair: a single-centre experience. Eur J Vasc Endovasc Surg. 2009;38:155-61.

21. Guillou M, Bianchini A, Sobocinski J, Maurel B, D'Elia P, Tyrrell M, et al. Endovascular treatment of thoracoabdominal aortic aneurysms. J Vasc Surg. 2012;56:65-73.

22. Lachat M, Veith FJ, Pfammatter T, Glenck M, Bettex D, Mayer D, et al. Chimney and periscope grafts observed over 2 years after their use to revascularize 169 renovisceral branches in 77 patients with complex aortic aneurysms. J Endovasc Ther. 2013;20:597.

23. Mastracci TM, Greenberg RK, Eagleton MJ, Hernandez AV. Dura-bility of branches in branched and fenestrated endografts. J Vasc Surg. 2013;57:926-33.

24. Moulakakis KG, Mylonas SN, Avgerinos ED, Kakisis JD, Brunkwall J, Liapis CD. Hybrid open endovascular technique for aortic thoracoabdominal pathologies. Circulation. 2011;124:2670-80.

25. Tshomba Y, Melissano G, Civilini E, Setacci F, Chiesa R. Fate of the visceral aortic patch after thoracoabdominal aortic repair. Eur J Vasc Endovasc Surg. 2005;29:383-9.

26. Perrin D, Demanget N, Badel P, Avril S, Orgéas L, Geindreau C, et al. Deployment of stent grafts in curved aneurysmal arteries: toward a predictive numerical tool. Int J Numer Method Biomed Eng. 2015;31:e02698.

27. Premprabha D, Sobel J, Pua C, Chong K, Reilly LM, Chuter TAM, et al. Visceral branch occlusion following aneurysmal repair using multibranched thoracoabdominal stent-grafts. J Endovasc Ther. 2014;21:783-90.

28. Kamohara K, Furukawa K, Itoh M, Morokuma H, Tanaka H, Hayashi N, et al. Evaluation of the optimal visceral branch configuration in open thoracoabdominal aortic repair by computed tomography. Ann Thorac Cardiovasc Surg. 2015;21:59-65. 